\title{
Appointment scheduling system in multi doctor/multi services environment
}

\author{
Ivica Lukić
}

J. J. Strossmayer University of Osijek, Faculty of Electrical Engineering, Computer Science and Information Technology Osijek Kneza Trpimira 2b, Osijek, Croatia ivica.lukic@ferit.hr

\section{Mirko Köhler}

J. J. Strossmayer University of Osijek, Faculty of Electrical Engineering, Computer Science and Information Technology Osijek Kneza Trpimira 2b, Osijek, Croatia mirko.kohler@ferit.hr

\section{Erik Kiralj}

J. J. Strossmayer University of Osijek, Faculty of Electrical Engineering, Computer Science and Information Technology Osijek Kneza Trpimira 2b, Osijek, Croatia

\begin{abstract}
Appointment scheduling systems are used by health care providers to manage access to their services. In this paper an algorithm and a web application for automatic appointment scheduling is presented. Both are implemented using the concept of booking appointments for patients for a specific service offered by each doctor. The purpose of the application is to make signing up for a specific service easier for patients and to improve health tourism in Croatia by maximizing doctor's efficiency and minimize patient waiting time. Medical providers are added to the system, they add the services which they provide, and each service offered has its own duration time. Users register, search for services matching their parameters, and schedule an appointment for the requested service. Available appointments are generated using the presented algorithm, which is the main part of this paper. The algorithm searches the database and returns possible appointments. If patient has more than one appointment, possible appointments time can be before the existing appointment, between two appointments, or at the end of the last appointment. Thus, web application enables the patient to reserve desirable appointment time.
\end{abstract}

Keywords - scheduling algorithm, health tourism, medical organization, web application

\section{INTRODUCTION}

Internet and mobile applications have become popular and part of our everyday life. Their aim is to make life much more comfortable by providing users with useful information and services in a transparent way. Thus, users do not need to worry about complicated calculations, configuration and scheduling tasks to get appointment for services that they need.

Organization of a medical appointment scheduling systems is a mandatory for an efficiency medical system. To improve healthcare tourism in Croatia, we must ensure timely access to health services for all patients. Timely access is a key factor for achieving successful medical results. It is also an important determinant of patient satisfaction. For a people, the biggest burden is making an appointment and going to doctor's office, because they know beforehand that they will be waiting. If they have more appointments, it is necessary to reduce or completely eliminate waiting time at medical institution. On the other hand, the doctors are busy people and want to use it efficiently. Every time that patient is late on appointment or just does not come, doctors schedule will be empty and unused. In this paper, we are focused to reduce doctor's idle and overtime.

Organizing an appointment in office with only one doctor is a complex task. First, one of the patient scheduling models must be selected [1-3]. However, without additional information, such as the patient's medical history, the patient's condition, whether it is a first or follow up visit, or some other reason, it is difficult to determine the duration of the examination. Consequently, organizing and ordering patients in a multi doctor/ 
multi services environment is more demanding task than in office with only one doctor.

The focus of this paper is to present algorithm for organization and automatic appointment scheduling of patients in multi doctor/multi services environment. It does not consider problems pertaining to the size of staff and facilities or with resource allocation in multiple-service-site systems like in [4]

\section{SCHEDULING ALGORITHM}

In this paper algorithm for automatic appointment scheduling in multi doctor/multi service environment is presented. We observed different approaches and combined them to get final algorithm which suits for such environment. Our algorithm uses the usual block schedule similarly as in $[5,6]$. In these studies, authors use analytical methods instead of simulation to estimate performance. Some other studies use a dynamic programming approach to determine the optimal variable-sized multiple-block schedule approaches [79]. Another study [10] suggests heuristic approach in which service time depends on the relative position of the service in the schedule. In other study [2] simulation-based techniques are used to measure the performance Appointment scheduling algorithm in health care using variety of heuristic appointment rules. Another approaches use genetic algorithms and machine learning for patient scheduling in highly constrained situations [11].

Our algorithm is combination of heuristic, dynamic programming and genetic approach. The construction of an automated scheduling algorithm is complex task because there is need to schedule human resources. But manual scheduling takes a lot of time and many administrative work and in some cases is impossible to find solution. The heuristic algorithms are used to find a solution close to the optimal in cases where finding the optimal solution is not possible. These algorithms work by getting closer and closer to the optimal solution as they progress. Their merit is that they can find a solution very close to the optimal solution in a relatively short time. Another approach is dynamic programming which simplifies a complicated problem by breaking it down into simpler sub-problems in a recursive manner. Sub-problems can be solved different programing algorithms.

Genetic algorithms are subgroup of heuristic algorithms and they are used to solve optimization problems. Genetic algorithms use idea of natural selection and genetic processes in the nature. As the nature life evolve using the principles of natural selection genetic algorithms also evolve to resolve problems. For the genetic algorithm knowledge structures, structural changes and the operators which change structure. To change knowledge structures different approaches can be used. The simplest approach parameters change and that changes system's behavior [11]. In our algorithm the most important parameters are the number of patients, number of medical staff, services and available appointments. These parameters determine scheduling quality:

- Appointment duration

- Doctor's idle time and overtime

- Patient's waiting time between appointments

- Total number of appointments is certain period

To improve scheduling quality appointment duration, doctor's idle time and patient's waiting time should be minimized, while the total number of appointments should be maximized. In this paper, parameter change is used for algorithm evolving. If the quality of service is reduced algorithm will change parameters to evolve. For example, if patient's waiting time is too long, the algorithm can reduce the number patients or add more medical staff to the system. If some doctors have many overtime, scheduling algorithm will transfer appointment to less busy doctor. There are many factors that impact on scheduling algorithm and it is impossible to apply them all.

The algorithm is presented using flowchart diagram which is consisted of a series of arrow-related symbols that define the flow and direction of program execution. This algorithm representation is simple, easy to review and easy to find. Problems can be easily analyzed and compared to another problem, which shortens the time to find a solution. In order to find available appointment time, it is necessary to create database for storing data. Database E-R diagram is shown in Fig. 1.

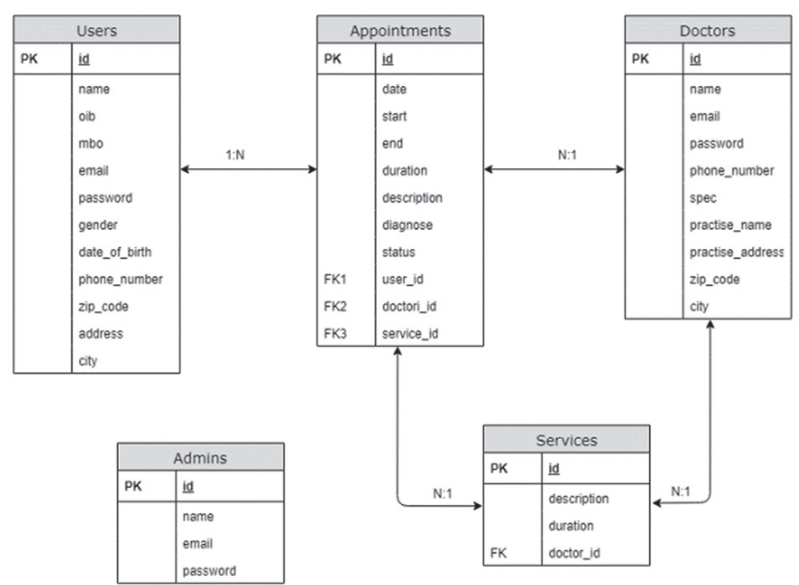

Fig. 1. Database E-R diagram

As shown in Fig. 1. database contains the following tables:

- Users represents the users (patients)

- Doctors represents the doctors

- Appointments represents the scheduled appointments of users with each doctor 
- Admins contains information about the application administrators

- $\quad$ Services is providing all possible services offered by individual doctors

User and Appointments tables are related by a 1: $\mathrm{N}$ relation, which means that one user can have more than one appointment, while one appointment only applies to one user (patient). Further, the Appointments and Doctors tables are related by a N: 1 link, indicating that one appointment can only refer to one doctor, while one doctor may have multiple appointments. The Appointments and Services table are related by a $\mathrm{N}$ : 1 relation, since one appointment can have only one service, while the same service can belong to several different appointments. In addition, the Doctors and Services tables are related by a N: 1 relation, indicating that one doctor may offer multiple services while one service belongs to only one doctor. The Admins table is not related with other tables, but has a separate role. Application is made using PHP framework named Laravel which uses migration to create the tables.

Our algorithm starts by looking for an already reserved appointments and makes them unavailable for future search. When a patient chooses the date range in which he or she would like to book an appointment, it is primarily followed by checking that the selected dates are valid. Also, algorithm searches for patient's already reserved appointments in selected time period to prevent appointment collision. The user can set the start date as early as tomorrow, while the end date could be same as start date, or any other date in future.

The next step is check for existed records in the Appointments table for the selected time period. If there are no records for the selected time period, the user is offered an appointment at the beginning of each day in selected time period. In this paper we used 08:00AM for the beginning of business day. Start time can be changed independently for any medical institution. If there is a record in the database for the selected dates, it is checked when first appointment starts for that day. If the start of the first appointment is greater than 08:00, it means that there are empty time slots before the first appointment of the day, and it is checked can be offered for appointment. In order to be able to offered, it is necessary to check whether the appointment time fits in the empty space until the next reserved appointment. If the condition is fulfilled appointment is reserved for current user.

In case these conditions are not fulfilled, and there is no free time slot before first reserved appointment, algorithm checks are there another free time slots for comparison in the database on the same day. If free time slot is found, it is checked whether the selected appointment ends before 16:00 hours, since it is assumed that is the end of working hours. End time of working hours also can be changed independently for any medical institution. If the condition is fulfilled, appointment is reserved for current user, and if not, it means that the day has been filled and appointment checking will be moved to the next date selected by the user.

Algorithm checks for the next date in selected time period and searches for the free time slot in the same way as for the first day in previous step. After all days are checked and algorithm did not find free time slot, appointment could not be reserved and algorithm has failed. The failure to meet this requirement means that there is not enough free time between start and end date, thus user is prompted to select another time period. The implementation of the algorithm is shown in Fig. 2.

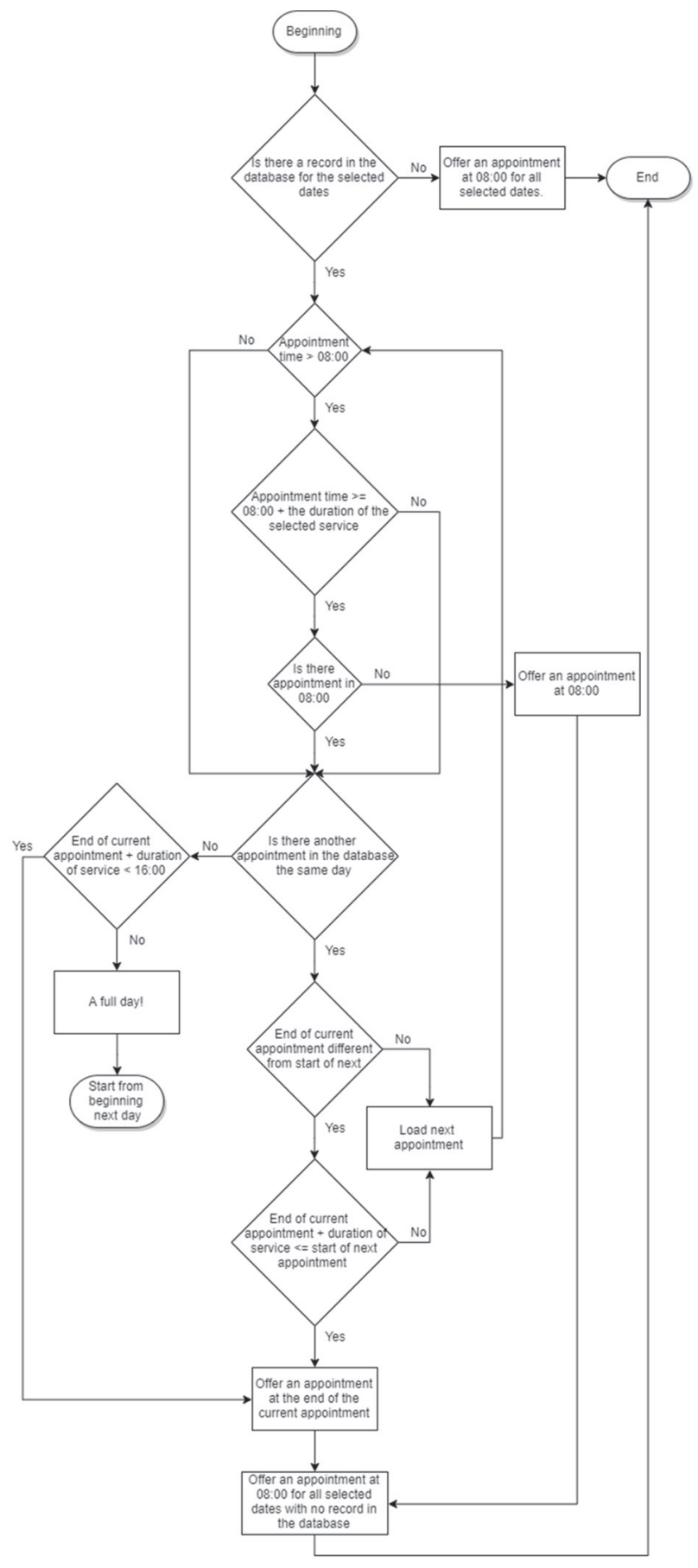

Fig. 2. Block diagram for appointment scheduling 


\section{APPLICATION}

The basic concept of the application is focused on health care tourism and goal is to achieve satisfaction of patients. Application should be user friendly and should ensure easy way to make the appointment. The patient selects the medical institute and the city in which he wants to make an appointment. After selection of medical institute or city all available medical staff are shown to the patient, and by selecting one, patient reserves one of the available appointments that suits him best. In Fig. 3. the homepage of the internet application is displayed.

If they want to book an appointment, patients need to log in with their user information. The patient is logged in by entering the Insured Identity Number and password. In case the patient does not have an account, registration is required. By choosing to register, the form is shown as in the Fig. 4.

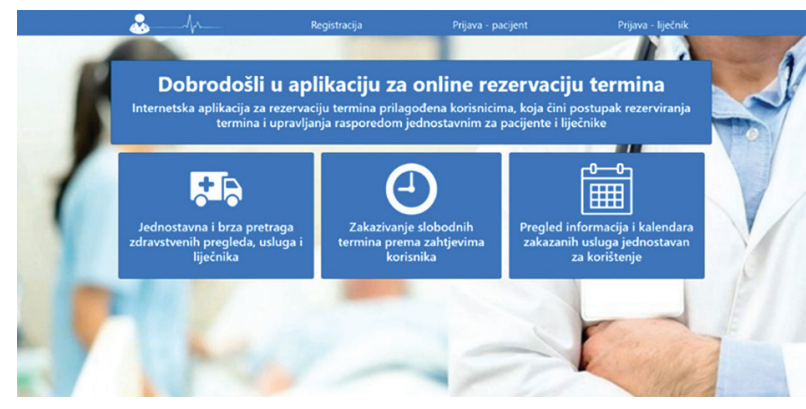

Fig. 3. Application home page

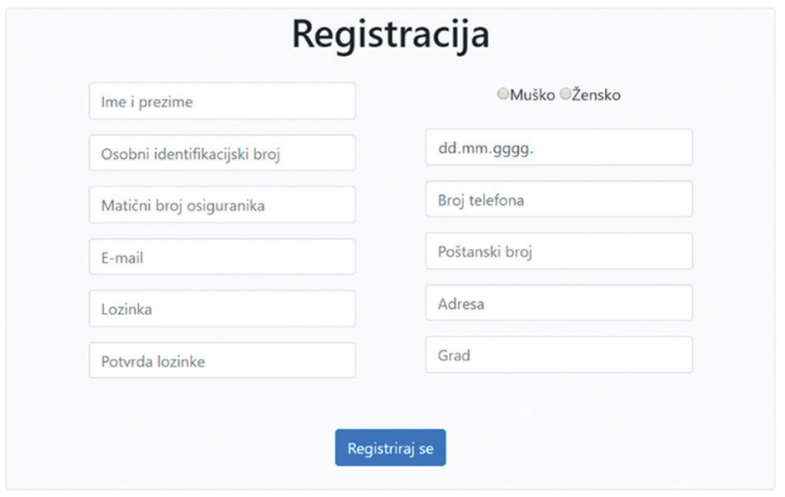

Fig. 4. Registration form

After registration user is redirected to user profile as shown in Figure 5.

Logged patient can search for the medical institution where the appointment (service) is going to be scheduled. The first thing the user chooses is the medical institution and the city where they want to book an appointment. In case the user's selection does not find any result, the user receives feedback and is offered a return to the homepage. In the next step all founded doctors are shown that fit the parameters and their basic information (first and last name, address, email, phone number, office address, etc.). The user selects the doctor who best suits his requirements. After the user has selected the doctor with whom he wants to request a service, then the service itself is selected. The user chooses the service for which he wants to book an appointment. Also, in this booking step patient will see a map with the exact location where the examining office is located as Shown in Fig. 6.
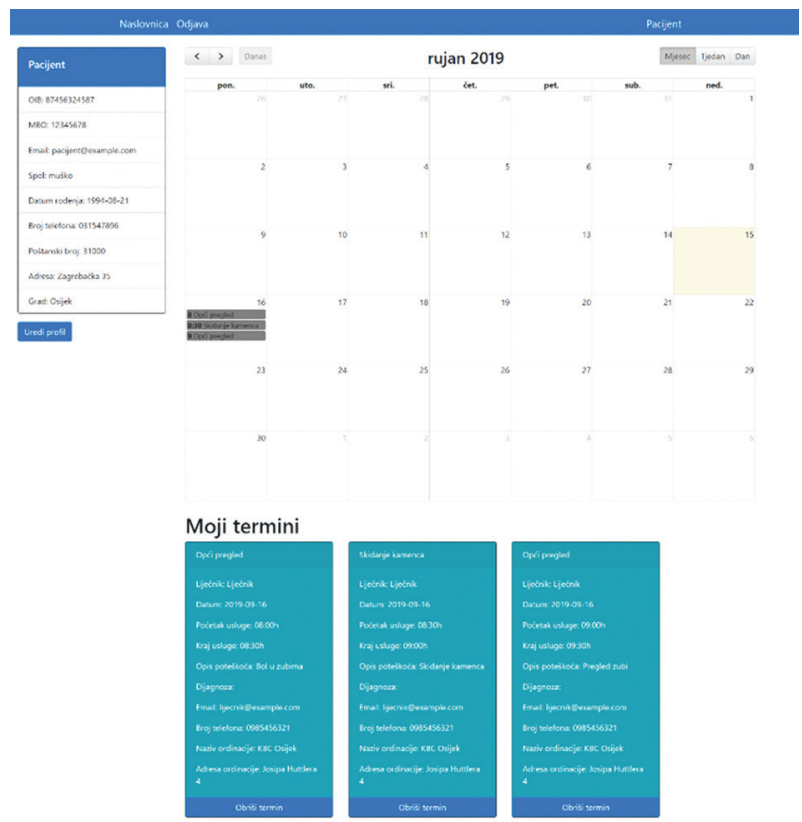

Fig. 5. Patient profile page

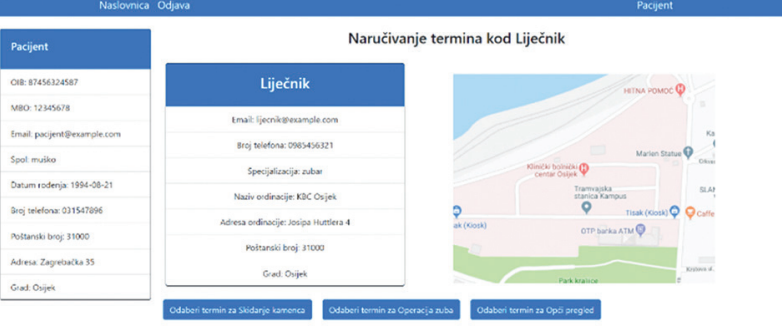

Fig. 6. Information about doctor and his location on map

In the next step, it is necessary to select the date range for which the patient wants to see the available appointments, and to choose the one that suits him best. The start date of the range must be one day longer than the current day, since it is not possible to book an appointment in the current day, but no earlier than the next day. In the event that this condition is not fulfilled, the same page is reloaded and the patient is informed to choose another time period. After selecting a valid date range, the algorithm performs its task and returns to the patient the appointment information and process is over.

In addition to being able to sign up for patients, there is also the option to sign up for medical staff who offer their services. The medical staff is not able to register himself, but they are added to the system by the application administrator. When an administrator enters 
a new medical staff into the system, they receive a welcome message at the email address with the user name and password that he logs in to. A password is randomly generated when creating account, and only the created account has access to the password. When a doctor or some other medical staff is logged in, a home page opens showing all the available terms of the registered doctor. As with patients, a calendar view of the appointment schedule is set. Below the calendar view, all the reserved appointments of the patients are listed in card form, where it is possible to see the details of the appointment. In addition to scheduling details, below the calendar is a form to add services that the doctor or some other medical staff offers. Selecting the "Add new service" option opens a page where the description of the service offered and the duration of the service itself can be entered. Successful entry reloads the profile listing the services offered. Each enrolled service can also be removed by selecting the "Delete Service" option, located at the end of the name of each service. Doctor's profile with all appointments and services offered are shown in Fig. 7.

In addition to reporting patients and doctors, there is also a way to sign up for a site administrator. Once logged in administrator can see all the medical staff in the database and their information. There are options for each doctor with which the administrator has the ability to edit information and remove the doctor from the database. In addition to existing doctors, the administrator also has the option "Add a new doctor", since doctors do not have the option to register with the system. The admin dashboard layout is displayed in Fig. 8.

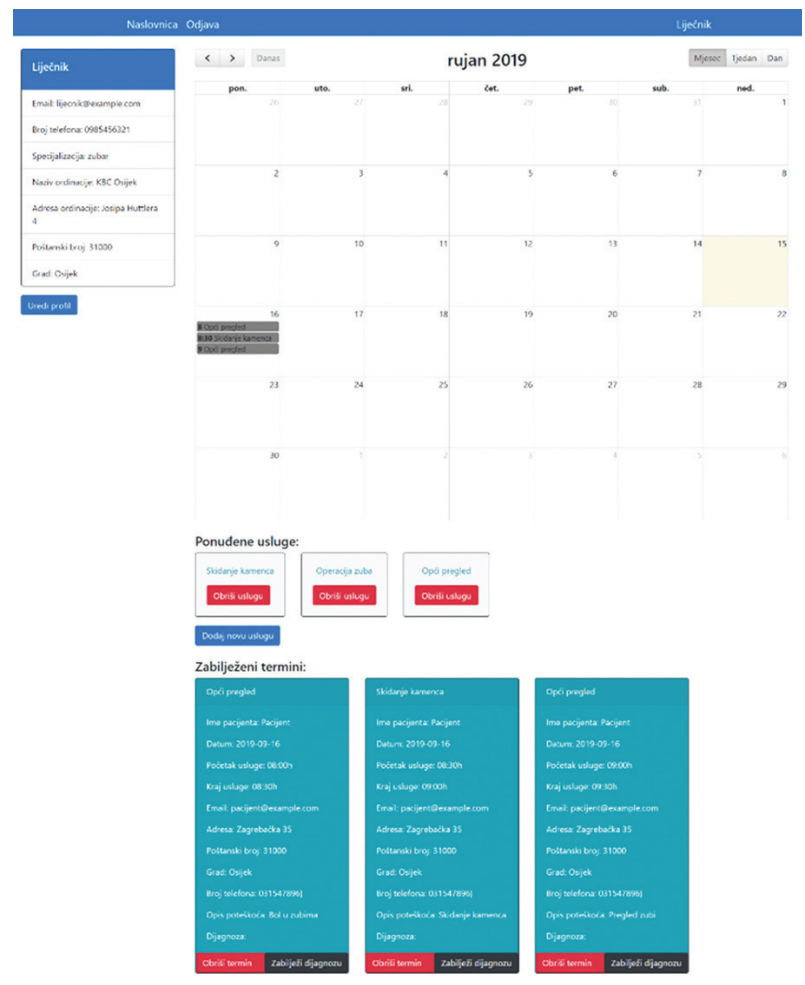

Fig. 7. Doctor profile page

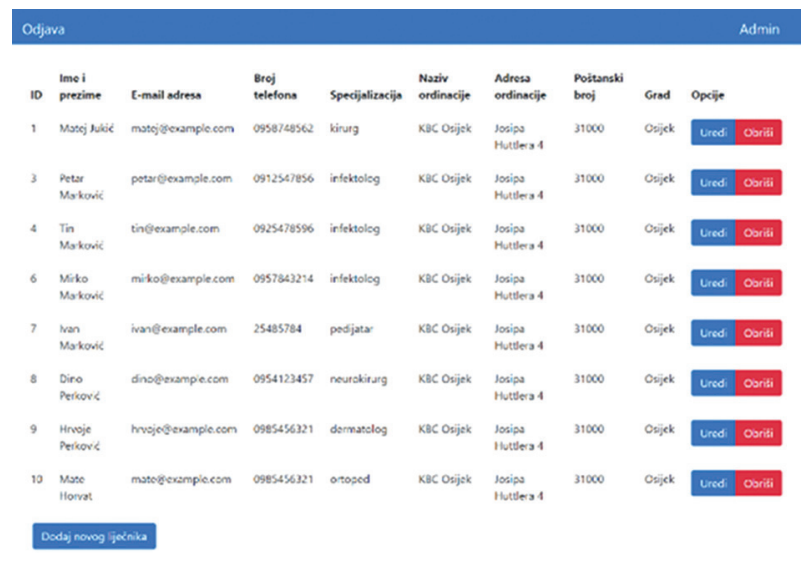

Fig. 8. Administrator profile page

\section{CONCLUSION}

In this paper an algorithm and online application for automatic appointment scheduling are presented. The application is implemented using the concept of booking patients' appointments for a specific service offered by individual medical staff. Primarily plan was to improve health tourism in Croatia by developing algorithm which will maximize doctor's efficiency and minimize patient waiting time. By maximizing efficiency patients will be more satisfied and have more services in selected time period. The algorithm is developed for that purpose and it allocates appointments to the database. The application itself is created in a Laravel which is used to build web applications based on MVC architecture. The layout scheme of the database was designed to contain all necessary data for the proper operation of the application. The main part of this paper is the development of the scheduling algorithm for multi/doctor and multi/patient system. The algorithm is designed to view the database and return an empty appointment which is appropriate for doctors and patients, remove overlaps and minimize waiting time. The application has two modes of user login, namely patients and doctors. Each has different functions and different views of the content, since they have different roles. Patients have been registered, logged in, selected, and scheduled appointments with the ability to edit their information, while doctors have been disabled from registering and added by the application administrator. Doctors have the ability to view scheduled appointments, add and delete individual services they offer, and edit their information. The response of the application is relatively fast, but after a while the amount of data in the database would increase and the speed would depend on the strength of the server. The problem could be solved by storing data in another memory location after a certain amount of time has elapsed since the end of the term. In future work we plan different improvements based on practical experience by using this application. We plan to assess doctors and patients to get feedback and find out possible improvements. 


\section{REFERENCES:}

[1] N. T. J. Bailey, "A study of queues and appointment systems in hospital outpatient departments, with special reference to waiting times", Journal of the Royal Statistical Society, No. 14, 1952, pp. 185-199.

[2] C. J. Ho, H. S. Lau, "Minimizing total cost in scheduling outpatient appointments", Management Science, Vol. 38, No.12, 1992, pp. 1750-1762.

[3] T. Cayirli, E. A. Veral, "Outpatient scheduling in health care: a review of literature", Production and Operations Management, No.12, 2003, pp. 519549.

[4] X. Chao, L. Liu, S. Zheng, "Resource allocation in multisite service systems with intersite customer flows", Management Science, Vol. 12, No. 49, 2003, pp. 1739-1752.

[5] M. White, M. Pike, "Appointment systems in outpatient clinics and the effect of patients' unpunctuality", Medical Care, No. 2, 1964, pp. 133-145.

[6] A. Soriano, "Comparison of two scheduling systems", Operations Research, No. 14, 1966. , pp. 388-397.
[7] E. L. Villegas, "Outpatient appointment system saves time for patients and doctors", Hospitals J.A.H.A., No. 41, 1967., pp. 52-57.

[8] E. Rising, R. Baron, B. Averill, "A system analysis of a university health service outpatient clinic", Operations Research, No. 21, 1973., pp. 1030-1047.

[9] B. Fries, V. Marathe, "Determination of optimal variable-sized multiple-block appointment systems", Operations Research, No. 29, 1981., pp. 324-345.

[10] L. W. Robinson, R. R. Chen, "Scheduling doctors appointments: optimal and empirically-based heuristic policies", IIE Transactions on Scheduling and Logistics, No. 35, 2003., pp. 295-307.

[11] V. Podgorelec, P. Kokol, "Genetic Algorithm Based System for Patient Scheduling in Highly Constrained Situations", Journal of Medical Systems, Plenum Press, Vol. 21, Num. 6, 1997., pp. 417-427.

\section{ACKNOWLEDGMENTS}

The paper is financed by project Optimization and scheduling in a dynamic system with multiple users and service providers. 